\title{
Atenção especializada ambulatorial no Sistema Único de Saúde: para superar um vazio
}

\author{
Specialized outpatient care in the Unified Health System: \\ how to fill a void
}

Charles Dalcanale Tesser ${ }^{1}$ Paulo Poli Neto ${ }^{2}$
${ }^{1}$ Departamento de Saúde Pública, Centro de Ciências da Saúde, Universidade Federal de Santa Catarina. Campus Universitário/ Centro de Ciências da Saúde, Trindade. 88040-900 Florianópolis SC Brasil. charles.tesser@ufsc.br

${ }^{2}$ Departamento de Saúde Comunitária, Universidade Federal do Paraná. Curitiba PR Brasil.

\begin{abstract}
The structuring of specialized outpatient care is a bottleneck in the operation of the Unified Health System. Based on a brief discussion about this void in an organizational model, we propose the federal induction of a format of specialized services from the experiences of Centers of Support for Family Health (NASF). They adapted matrix operations and constitute an excellent prototype for the organization of specialized outpatient care. It allows for equal access and maximum proximity to the specialized care of the reality of primary care users, the personal relationship and the close relationship between the family health teams and medical and non-medical specialists, enabling mutual lifelong learning, negotiated regulation and increased efficacy of primary care. Municipal experiences of Florianopolis and Curitiba are synthesized as partial examples of the proposal. the structure of care in mental health of Florianópolis, all organized as a matrix support is briefly described; and we focus on the change in the action of the support teams of Curitiba, which gradually began to engage, involve and mediate the relationship between basic and specialized care. This format can be expanded to most medical specialties.
\end{abstract}

Key words Unified Heath System (SUS), Secondary care, Health policies, Health services planning
Resumo A estruturação da atenção especializada ambulatorial é um gargalo na construção do Sistema Único de Saúde. A partir de breve discussão desse vazio de um modelo organizativo, propomos a indução federal de um formato de serviços especializados a partir das experiências dos Núcleos de Apoio à Saúde da Família (NASF), cuja atuação matricial adaptada é um protótipo ótimo de organização do cuidado especializado ambulatorial. Ela permite a equidade no acesso e a máxima proximidade do cuidado especializado da realidade dos usuários, o relacionamento personalizado e a articulação intima entre as equipes de saúde da família e os especialistas, viabilizando educação permanente mútua, regulação negociada e aumento da resolubilidade da atenção básica. Aspectos das experiências municipais de Florianópolis e Curitiba são sintetizadas como exemplos parciais da proposta. É brevemente descrita a estruturação do cuidado em saúde mental de Florianópolis, toda organizada como apoio matricial; e a mudança de enfoque das equipes de apoio de Curiti$b a$, que progressivamente passaram a sem empenhar, envolver e mediar a relação entre a atenção básica e a especializada. Este formato pode ser adaptado e expandido para a maioria das especialidades médicas.

Palavras-chave Sistema Único de Saúde, Atenção secundária, Políticas de saúde, Planejamento de serviços de saúde 


\section{Introdução}

O Sistema Único de Saúde (SUS) vem sendo construído com algumas vitórias. Efetivou-se exitosa indução federal de expansão e estruturação da atenção primária à saúde (APS), via Estratégia Saúde da Família (ESF). Uma APS qualificada oferece cuidado profissional (generalista) abrangente com acesso universal facilitado, acompanhamento da pessoa ao longo do tempo (longitudinalidade), além de filtragem das necessidades, acionamento e coordenação dos cuidados especializados prestados por outros profissionais e serviços do sistema de saúde quando necessário ${ }^{1,2}$.

Mesmo sendo pequena minoria, os problemas em que há necessidade de profissionais especializados (doravante chamados apenas de especialistas, médicos e de outras profissões, por contraste com os profissionais generalistas que atuam na APS atendendo a todos os tipos de problemas de saúde) não são raros e são situações em que há maior gravidade clínica e ou maior dificuldade diagnóstica. O cuidado especializado é necessário para dar efetividade e consequência à APS e prover assistência especializada aos que dela necessitam, complementando-a, para que o Sistema aproxime-se de uma integralidade desejada.

Um dos grandes problemas do SUS, reconhecido também pelo governo federal ${ }^{3}$, é a estruturação "insuficiente" e heterogênea da rede de cuidados especializados, tornando-a um gargalo na construção do Sistema ${ }^{4,5}$. A normatização do SUS atribui aos municípios a responsabilidade pela rede ambulatorial especializada, permitindo associações de municípios. O Ministério da Saúde (MS) repassa recursos aos municípios para os procedimentos e as consultas especializadas realizadas, além de transferir recursos relativos aos Núcleos de Apoio à Saúde da Família (NASFs), induzidos por financiamento federal desde $2008^{6}$. O MS instituiu os NASFs em uma proposta de apoio matricial para as equipes da ESF. Projetou neles ações interdisciplinares, de apoio (discussões de casos e projetos terapêuticos) e outras típicas da ação generalista da APS (promoção, prevenção, planejamento), sem valorizar sua atuação assistencial como referência especializada, embora não a proíba ${ }^{6,7}$. Além disso, os NASFs só podem ter uma mínima parte das especialidades médicas: clínica médica, pediatria, ginecologia, psiquiatria, geriatria, homeopatia e acupuntura ${ }^{6}$.

Os serviços especializados existentes no SUS comumente organizam-se em ambulatórios, onde especialistas recebem pacientes através de encaminhamento ou referência, sem conhecer os profissionais que encaminharam e sem acesso aos prontuários com registros do cuidado previamente realizado ${ }^{8}$ (salvo quando há prontuários eletrônicos acessíveis). Quase sempre esse trabalho é isolado da APS 9 : os especialistas tendem a criar um vínculo assistencial solitário; há frequentemente retenção desnecessária de pacientes e geração de filas de espera para especialistas, dificultando o vínculo com a APS e a coordenação do cuidado.

O Ministério da Saúde limitou-se a editar diretrizes para a organização da Rede de Atenção à Saúde (RAS) do SUS ${ }^{10}$. Embora seja um avanço significativo, não significaram uma proposta concreta e operacional e um investimento federal indutivo de sua construção. Elas são convergentes, em geral, com a proposta de RAS sistematizada por Mendes", que a define como "organizações poliárquicas de conjuntos de serviços de saúde, vinculados entre si por uma missão única, por objetivos comuns e por uma ação cooperativa e interdependente, que permitem ofertar uma atenção contínua e integral a determinada população, coordenada pela atenção primária à saúde [...]". O cuidado especializado ambulatorial, objeto de nosso interesse, pode ser considerado grosso modo como atenção secundária, que Mendes ${ }^{9}$ considera não necessitar estar tão concentrado espacialmente como os pontos de atenção terciária. Todavia, suas propostas de RAS não operacionalizam a construção progressiva dela, nem especificam os modos operativos de organizar as relações entre os especialistas e os generalistas da APS, embora afirmem a necessidade de comunicação contínua e de coordenação pela APS de forma territorializada e personalizada.

Mais de 25 anos após a formalização legal do SUS, não há diretrizes operacionais claras e recursos substanciais para induzir a ampliação e a estruturação da atenção especializada que desviem das mazelas acima mencionadas. Existem experiências municipais em situações específicas, como a gestação de risco, com mais relação entre a APS e a atenção especializada, com protocolos para encaminhamento e referências regionais ${ }^{9,11}$. Outras iniciativas tentam evitar a remuneração de serviços especializados por procedimentos, contratando pacotes de atenção a problemas específicos, ou criando ambulatórios especializados com servidores assalariados (com as dificuldades citadas). Tais iniciativas não constituem um formato organizativo de serviço especializado que possa ser induzido nacionalmente, analo- 
gamente à indução federal das equipes de SF que gerou a ESF, hoje padrão-ouro da APS brasileira, cujo sucesso triplicou-a.

O presente artigo discute este gargalo (o relativo vazio da atenção especializada no SUS), esboçando algumas interpretações históricas, apenas o suficiente e de forma superficial para contextualizar a defesa da necessidade de elaboração e indução de um formato organizacional de serviços de cuidado especializado no SUS, a ser construído nos municípios (ou entre eles, em regiões), a partir de experimentações institucionais municipais em andamento. Após a proposição de um ponto de partida que é a experiência dos Núcleos de Apoio à Saúde da Família (NASF), esboçamos as bases de uma proposta de indução federal de organização do cuidado especializado, ainda pouco elaborada, mas por hipótese potente e promissora enquanto ideia reguladora. Como base empírica parcial da proposta, sintetizamos aspectos de duas experiências municipais brasileiras que avançam no sentido proposto.

\section{Um vazio a ser compreendido, enfrentado e superado}

A atenção especializada no SUS constitui-se em gargalo por variadas razões. Após a ditadura militar, com privatização de recursos públicos para construção de hospitais privados; a crítica da medicalização social; as análises marxistas da medicina e da formação médica; as críticas ao biologicismo mecanicista e à fragmentação do cuidado; as discussões sobre determinação social da saúde-doença e promoção da saúde; após tudo isso parece compreensível haver uma 'aversão’ na Saúde Coletiva em relação à medicina especializada. Mas isso tem potencial interpretativo pequeno, porque a atenção superespecializada (transplantes, hemodiálises, cirurgias cardíacas, quimioterapias, etc.) é forte e organizada no SUS; o que nos remete a outras razões.

Após a formalização do SUS, houve uma forte hegemonia do neoliberalismo no país, com pouca concretização operacional de seu ideário $^{12,13}$. Nesse contexto adverso, conseguiu-se iniciar a organização do SUS sem grandes reformas $^{14}$, pelos seus dois extremos: o alto custo e a APS. Poucos serviços privados querem ou podem oferecer os tratamentos de alto custo e pouquíssimos cidadãos dentre os que deles necessitam os poderiam pagar. Por seu turno, a APS tem baixo impacto mercadológico, seus usuários são suficientemente pobres para não usarem o sistema privado. A essa maioria pobre coube a APS, en- quanto os cuidados especializados permaneceram na maior parte na iniciativa privada ${ }^{15}$.

Esses percalços políticos sugerem uma hipótese que ilumina a persistência do vazio: uma organização da atenção especializada no SUS seria muito competitiva e mesmo avassaladora para o mercado da saúde. Provavelmente, poucas pessoas pagariam planos de saúde se acessassem cuidado especializado quando necessário em tempo adequado no SUS. Isso impactaria enormemente a medicina privada no Brasil, abundante e mal distribuída ${ }^{16}$.

É grande a dificuldade política e operacional dessa empreitada: o cuidado médico especializado e a sua formação (residências médicas) têm sido regulados e exercidos pelas corporações médicas especializadas e por (supostas) regras do mercado. Há quatro vezes mais médicos no setor privado que no SUS, sendo que no país 55\% têm título de especialista, mas somente $1 \%$ em medicina de família e comunidade ${ }^{16}$.

Outros aspectos ainda merecem consideração. Um é a herança cultural e ideológica brasileira sobre a APS, vista como serviços de saúde pública de prevenção, educação e vigilância, com atuação clínica apenas em situações epidemiologicamente relevantes ${ }^{17}$. Houve alguma mudança desse ideário na direção da promoção da saúde ${ }^{18}$, na qual persiste, porém, um afastamento da clínica do adoecido. Tal visão, associada à 'aversão' à clínica e à busca por atuação nos determinantes sociais $^{19}$, desvia as discussões da Saúde Coletiva do cuidado clínico, tanto no ambiente da APS ${ }^{20}$ quanto, provavelmente, na estruturação do cuidado especializado no SUS, inibindo-a.

Além disso, a grande maioria dos profissionais, acadêmicos, gestores e servidores públicos em geral e do SUS não usam a $\mathrm{APS}^{20}$ nem os serviços especializados do SUS, pois têm acesso direto ao cuidado médico especializado privado, através de planos privados subsidiados ${ }^{21}$ e renúncia fiscal ${ }^{22}$. Há, portanto, dificuldades significativas, poderes políticos e econômicos antagônicos, conflitos de interesses e vícios ético-políticos sustentando a tolerância ao vazio de diretrizes inovadoras para a organização e a ampliação dos serviços de cuidado especializado dentro do SUS.

\section{Direção promissora}

A construção da universidade e integralidade implica incorporar o problema do cuidado especializado à pauta da Saúde Coletiva e do SUS, e construir um formato de 'boa organização' desse cuidado. Nesse sentido, há experiências pioneiras 
através dos NASFs, pouco exploradas no seu potencial de provisão de cuidado especializado.

A prática clínica especializada foi pensada no apoio matricial no artigo pioneiro de Campos, de $1999^{23}$. Ali, o matriciamento é proposto acrescido às funções assistenciais dos especialistas: trata-se de dar apoio técnico-pedagógico a equipes de referência (equipes de saúde da família [SF]), além de oferecer ações de cuidado especializado, reforçando os vínculos dos usuários com a equipe de SF. Em formulações posteriores ${ }^{8,24,25}$, há atenuação dessa função de provisão de cuidado clínico especializado: o apoio matricial é considerado "complementar aos mecanismos de referência e contrarreferência, protocolos e centros de regulação" ${ }^{8}$, mas a função assistencial continua presente genericamente. Já na diretriz federal para atuação dos NASFs ${ }^{6}$, contrariamente, sua função assistencial especializada diretamente ao usuário individual foi despriorizada ${ }^{7}$.

A atuação dos matriciadores de diversas profissões e especialidades constitui um modo de organização de fluxos negociados e equitativos ao cuidado especializado e de seu exercício. Essas experiências - espalhadas pelo país e ainda não avaliadas sistematicamente quanto a este aspecto - sugerem que as atuais equipes matriciadoras dos NASFs podem e devem ser progressivamente fundidas com a atenção especializada, absorvendo as atribuiçõos desta última. Equipes análogas aos NASFs com os demais especialistas médicos poderiam ser montadas, de modo que todos os especialistas (médicos e de outras profissões) poderiam atuar, na medida do possível e com adequações, de forma personalizada e regionalizada, matriciando um conjunto de equipes de SF, atendendo a população filtrada em corresponsabilização. Tal comunicação, adscrição e longitudinalidade facilitariam o conhecimento entre especialistas e generalistas da APS, com melhora da educação permanente mútua e discussões dos casos limítrofes e duvidosos (também por telefone ou via digital), avançando na superação da tradição viciosa dos ambulatórios especializados isolados e suas mazelas mencionadas, viabilizando coordenação do cuidado. Isso incide diretamente no centro das buscas por adequação do cuidado especializado, gerando um continuum coordenado e colaborativo de cuidados ${ }^{26}$, procurado nos sistemas de saúde ${ }^{27-30}$.

A função de coordenação do cuidado da APS tem estado cada vez mais forte e importante nas reformas europeias da APS $^{27,28}$, aproximando-se de forma convergente do apoio matricial brasileiro. A troca de conhecimentos e a colaboração entre generalistas da APS e especialistas vêm sendo propostos e realizados em vários países como estratégia de ampliar e qualificar o cuidado, com diferentes nomes, como colaborative care $^{31}$ e shared care $^{32,33}$.

Shared care, muito usado em situações crônicas, é definido como a participação conjunta de profissionais da APS e especialistas no planejamento e execução de cuidados a pacientes crônicos, informada por uma maior troca de informações, para além dos informes de referência e contrarreferência ${ }^{34}$. Os ingredientes principais para a efetivação do cuidado compartilhado, para Kelly et al. ${ }^{35}$, são: a) aproximação sistemática para o envolvimento dos serviços generalistas com os de especialidade; b) elaboração de um modelo coerente com as necessidades da população-alvo; c) um acordo que contemple a possibilidade de encontros para a revisão dos casos, quando necessário; d) atenção para seleção e contratação de pessoal e garantia de espaços de supervisão clínica para apoiar o desenvolvimento de habilidades e manutenção do modelo; e) fortalecimento das instâncias de gestão da clínica. Em meta-análise sobre o cuidado compartilhado, Foy et al. ${ }^{36}$ detectaram a comunicação interprofissional como principal fator associado à sua efetividade.

Oliveira e $\mathrm{Campos}^{26}$ analisaram artigos, revisões e experiências no Canadá, Espanha, Austrália, Reino Unido e Irlanda afirmando convergência na valorização da necessidade de cuidados colaborativos ou compartilhados. Segundo esses autores, a proposta de apoio matricial brasileiro vai além das recomendações da literatura internacional, na proposição de: a) personalização das relações interprofissionais, b) contratualização da organização entre equipes com liberdade para adequá-las ao contexto; c) definição clara de responsabilidade e d) utilização de formas de cogestão.

A proposta de Mendes ${ }^{9}$ também é convergente ao afirmar que "os especialistas, no modelo de RAS, cumprem, em geral, o papel de interconsultor e de suporte para as ações da equipe de APS. As evidências demonstram que a atuação dos especialistas só agrega valor para as pessoas quando eles se conhecem pessoalmente e trabalham conjuntamente com os generalistas".

Também Terraza Núñez et al. ${ }^{37}$ defendem que para coordenar processos complexos nos quais intervêm diferentes serviços e profissionais, com volume de informação processada elevado (complexificados pela multiculturalidade e as precárias situações socioeconômicas da maioria da população brasileira), os melhores arranjos 
institucionais de coordenação do cuidado são os que se baseiam na adaptação mútua e no contato pessoal direto, viabilizando comunicação ágil (telefone, correio eletrônico, reuniões informais), grupos de trabalho interdisciplinar (discussões de casos, regulação negociada), profissionais, situações e mecanismos de enlace entre os profissionais dos diversos serviços e níveis assistenciais, estruturas matriciais, etc. Isso é o que vem sendo experimentado pelo apoio matricial dos NASFs, reconhecido como uma inovação na integração entre APS e cuidado especializado ${ }^{38}$, enquanto mudanças nas práticas já estão aos poucos sendo experimentadas. Como ilustração e exemplo prático, sintetizamos aspectos de duas experiências que exploram esse potencial.

\section{Atenção especializada em Saúde Mental via matriciamento em Florianópolis}

Florianópolis, que tem toda a APS estruturada pela ESF (com cobertura de $100 \%$ da população), organizou a assistência especializada ambulatorial em Saúde Mental (SM) centrada no apoio matricial ${ }^{39}$. Nesse muncípio, os profissionais de saúde mental estão há vários anos trabalhando incorporados aos NASF ou compondo as equipes dos Centros de Atenção Psicossocial (CAPS). As equipes de SM dos NASF (psiquiatras e psicólogos) se corresponsabilizam pelos casos filtrados e estruturam sua atuação conforme a necessidade das diversas equipes de SF.

Saraiva e Cremonese ${ }^{40}$ descrevem a construção da experiência: os casos que são atendidos pela equipe de saúde mental são decididos em conjunto com a ESF em discussões de caso de periodicidade combinada com a equipe de SF. Se a equipe, antes da reunião de matriciamento, tiver alguma dúvida importante ou quanto à necessidade de atendimento de urgência por profissional de SM, discute o caso por telefone com o profissional do NASF, para orientação e conduta. A escolha e o planejamento dos atendimentos grupais em SM são realizados com as equipes de SF considerando as necessidades prioritárias de saúde mental do território. O NASF faz a articulação entre os centros de saúde e os CAPS, organizando o fluxo e o processo de trabalho. Para isso, conta com reuniões distritais de saúde mental, mensais, com participação de profissionais de saúde mental dos NASF e dos CAPS, para discussão de casos, projetos terapêuticos e fluxos entre os serviços. Os casos a serem atendidos nos CAPS devem ser preferencialmente encaminhados pelas equipes de SM dos NASF. Os
CAPS também dão retaguarda às equipes de SF e NASFs, nas suas especificidades, assessorando em demandas específicas. Os indivíduos que buscam os CAPS por demanda espontânea, são acolhidos e avaliados por um dos membros da equipe; se não tiverem necessidade de acompanhamento nesse serviço, são atendidos pela ESF na sua área de residência, com apoio do NASF e das equipes dos CAPS. Os usuários acompanhados pelo CAPS que, após estabilização, recebem alta, são discutidos nas reuniões entre CAPS e NASF, para passarem a ser atendidos por estas equipes, sempre mantendo o vínculo com a ESF. Para facilitar esse fluxo, é imprescindível a existência dos espaços de discussão descritos entre a ESF, o NASF e o CAPS. Garante-se assim a construção de projetos terapêuticos singulares, realizada em conjunto nas diversas esferas da rede, e a conexão dos serviços e profissionais em uma linha de cuidado em saúde mental ${ }^{39,40}$.

Como descrito acima, em tal experiência há uma rotina periódica (negociada entre as equipes, em geral quinzenal ou mensal) de discussão permanente dos casos eleitos como necessitados de atendimentos especializados em SM e um suporte telefônico em casos de urgências e intercorrências para as equipes de SF pelo NASF, com o apoio se necessário do CAPS. Há uma regulação negociada personalizadamente entre equipe de SF e especialistas em SM do NASF, que atuam nos próprios serviços de APS regionalizadamente, tendo-se abolido completamente as listas de espera (antes existentes e demoradas) de encaminhamento para a saúde mental (psiquiatria e ou psicologia $)^{40}$. Todos os casos e demandas de cuidado especializado em SM são resolvidos nas reuniões de matriciamento e ou com conversas e negociações diretas (casos urgentes e duvidosos) com os apoiadores do NASF ou do CAPS. Os atendimentos individuais com especialistas em SM (psicólogos e psiquiatras) são viabilizados se julgados necessários, embora haja certa restrição de agenda para psicólogos e pressão para tratamentos psicológicos grupais ${ }^{41}$. Embora desconheçamos avaliações sistematizadas da experiência, é fato consensual na ESF local que a retaguarda especializada em SM foi viabilizada satisfatoriamente, enquanto formato organizativo institucional, pelas equipes matriciais - que podem realizar práticas ambulatoriais, consistindo em um exemplo parcial (restrito aos profissionais da SM) da ideia e proposta aqui defendida. A experiência tem limites, como a dificuldade de viabilizar tratamentos singularizados não farmacológicos como primeira abordagem e subexplo- 
ração de outros recursos comunitários e institucionais ${ }^{41}$, denotando restrições na concretização dos princípios da reforma psiquiátrica, mas é um exemplo exitoso indiscutível da viabilidade da construção de retaguarda especializada via apoio matricial.

Práticas de assunção do cuidado especializado (assumir ser referência especializada) pelos profissionais do matriciamento em saúde mental (em corresponsabilização e cuidado compartilhado com os profissionais generalistas da APS) são mencionadas em vários trabalhos e experiências municipais em todo o país ${ }^{42-49}$ e provavelmente são as mais avançadas experiências de provisão de cuidado especializado via matriciamento no SUS.

\section{Regulação e NASF em Curitiba}

Desde o início da década de 2000, Curitiba passou a contar com alguns profissionais que realizavam apoio in loco às equipes das Unidades básicas de saúde (UBS). Em 2009 foram constituídas equipes multiprofissionais (psicólogos, farmacêuticos, fisioterapeutas, nutricionistas e profissionais de educação física) que apoiavam um grupo de UBS. Por atuarem tanto em UBS tradicionais quanto em UBS com saúde da família foram chamados de Núcleos de Apoio à Atenção Primária à Saúde (NAAPS) ${ }^{50}$.

A tônica do trabalho desses profissionais eram as atividades educativas, geralmente com vários profissionais simultaneamente. Boa parte da carga horária era dedicada a grupos de gestantes, hipertensos e diabéticos, com orientações sobre mudanças de estilo de vida. Havia atividades de cuidado clínico focadas em alguns programas, como o da oxigenioterapia a cargo do fisioterapeuta e o do fornecimento de leites especiais com o nutricionista ${ }^{50}$. Os profissionais dos NAAPS tinham um papel quase nulo na regulação clínica para suas especialidades, como os da fisioterapia, psicologia ou nutrição. A consulta clínica individual era fortemente desestimulada. Em Curititba, nessa década, as UBS foram diminuindo sua capacidade produtiva, ofertando menos vagas para as consultas do dia, realizando menos procedimentos como pequenas cirurgias, suturas e concentraram progressivamente seu trabalho nos pacientes agendados com problemas específicos ${ }^{51}$.

A partir de 2013 houve mudança na orientação da gestão, no sentido de fortalecer o papel da APS: melhorar o acesso às consultas médicas, de enfermagem e odontológicas independentemen- te do problema de saúde ${ }^{52}$; oferecer uma carteira de serviços mais ampla ${ }^{53}$; filtrar melhor e acompanhar mais de perto os pacientes que precisam de atenção especializada.

Os NAAPS foram rebatizados de NASF e passaram por uma transformação, acompanhando a mudança na $\mathrm{APS}^{54}$. Os profissionais já participantes (em especial a fisioterapia, a psicologia e a nutrição) foram orientados, inicialmente por meio de encontros para discussão e de recomendações dos gestores municipais, regionais e locais, a avaliar as filas para especialidades da sua área, identificar os casos que poderiam ser atendidos na própria UBS e a atendê-los individualmente ou a discutir o caso com as equipes da APS. Em relação às atividades educativas, a orientação é a de centrar sua presença naquelas em que há a necessidade do seu conhecimento específico e, nas demais, procurar envolver outros profissionais que possam conduzir os grupos. Sugeriu-se evitar a participação de mais de uma categoria profissional de nível superior em atividades educativas que prescindam do saber específico desses profissionais. Publicou-se em janeiro de 2014 uma normativa que destaca o papel dos profissionais dos NASF na regulação clínica, na avaliação das filas de especialidades e no apoio às dúvidas dos profissionais das UBS ${ }^{55}$. É possível observar alguns resultados, como o aumento do número de atendimentos individuais das categorias não médicas dos NASF (Gráfico 1).

Até 2012 não havia em Curitiba nenhuma forma de regulação ou de avaliação das filas de encaminhamento para especialidades. Os médicos das UBS optavam ao encaminhar se o caso era ou não prioritário. Os casos prioritários passavam na frente dos demais automaticamente, sem avaliação de um regulador. Com o tempo, a maioria dos encaminhamentos era classificada como prioritária. Apesar de o município ofertar mais de 60 mil consultas especializadas ao mês, mais de $1 / 3$ do total de consultas médicas da APS, longas filas foram se formando ${ }^{56}$.

Somente em 2013, por um decreto municipal, inclui-se a categoria médica nos NASFs. Em julho de 2014 são compostas equipes do NASF com pediatras, ginecologistas, psiquiatras, médicos com atuação em geriatria e em clínica/ infectologia. A proposta é a de que esses profissionais atuem fortemente voltados para a regulação clínica, sendo a primeira referência para os médicos das UBS em relação às dúvidas da sua área de atuação, atendendo ombro-a-ombro ou individualmente pacientes e encaminhando para os ambulatórios secundários e terciários quando 


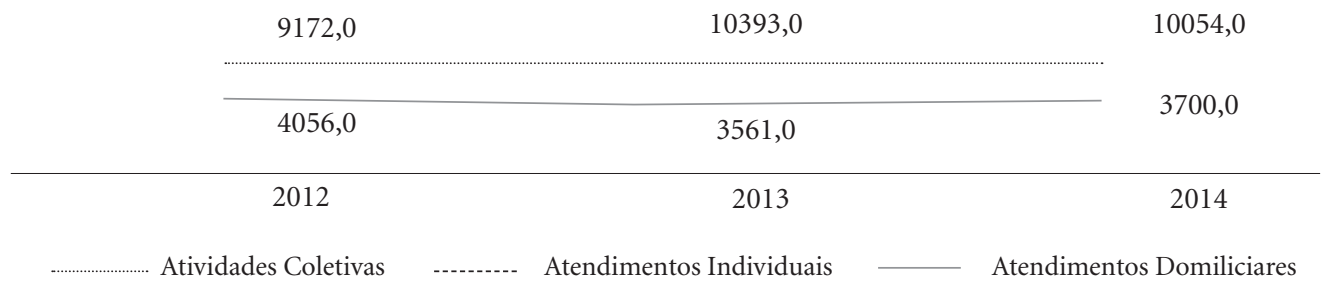

Gráfico 1. Evolução dos atendimentos individuais, coletivos e domiciliares dos NASF (pessoas atendidas) Categorias não-médicas, Curitiba, 2012-2014.

Fonte:Relatório Gerencial: Mapa Geral Dinâmico. Secretaria Municipal de Curitiba. 2015

necessário. Paulatinamente, esses profissionais dos NASF, médicos e não médicos, têm auxiliado a gestão municipal a definir qual a necessidade de serviços especializados, justamente por fazerem a ponte entre a APS e os demais níveis de atenção.

O formato organizacional de regulação clínica e de relação entre a APS e a atenção especializada que tem sido desenhado desde 2013 possui vários pontos de regulação e de telerregulação/ teleconsultoria (Figura 1).

Nesse desenho, o médico da APS teria no NASF e em segundas opiniões, via teleconsultoria/telerregulação, profissionais que responderiam presencialmente, por telefone, e-mail, aplicativos de celular ou pelo próprio prontuário eletrônico (que está sendo adaptado para permitir esse uso), respostas rápidas às suas dúvidas clínicas. Os profissionais dos NASF ou aqueles em ambulatórios especializados poderiam responder aos encaminhamentos/dúvidas da APS de diferentes maneiras: retorno da dúvida com orientações para manejo clínico; retorno com solicitações de mais informações da história, do exame físico, etc.; agendamento de consulta com o próprio teleconsultor; agendamento em outros serviços. Houve algum impacto nas filas para es- pecialidades, pequeno devido ao grande passivo dos anos anteriores (Tabela 1).

\section{Considerações finais}

As equipes dos NASFs reúnem no seu modus operandi ótimas características de um formato organizativo preliminar ou protótipo de serviço de atenção especializada, desde que atuem como tal, sem deixar de realizar matriciamento, e sejam dimensionadas e compostas adequadamente. Os NASF têm sido em muitos locais subexplorados como equipes assistenciais de retaguarda especializada, devido às diretrizes oficiais focadas no apoio e em ações generalistas de promoção e prevenção da APS, subutilizando a competência nuclear dos profissionais matriciadores. Por outro lado, em locais em que eles atuam somente com assistência especializada, ocorre o inverso e aí devem fazer também apoio técnico às equipes de APS, melhorando sua resolubilidade e contribuindo na sua educação permanente, concretizando o matriciamento ${ }^{7}$.

Todos os serviços especializados, na medida do operacionalmente possível, deveriam ter 


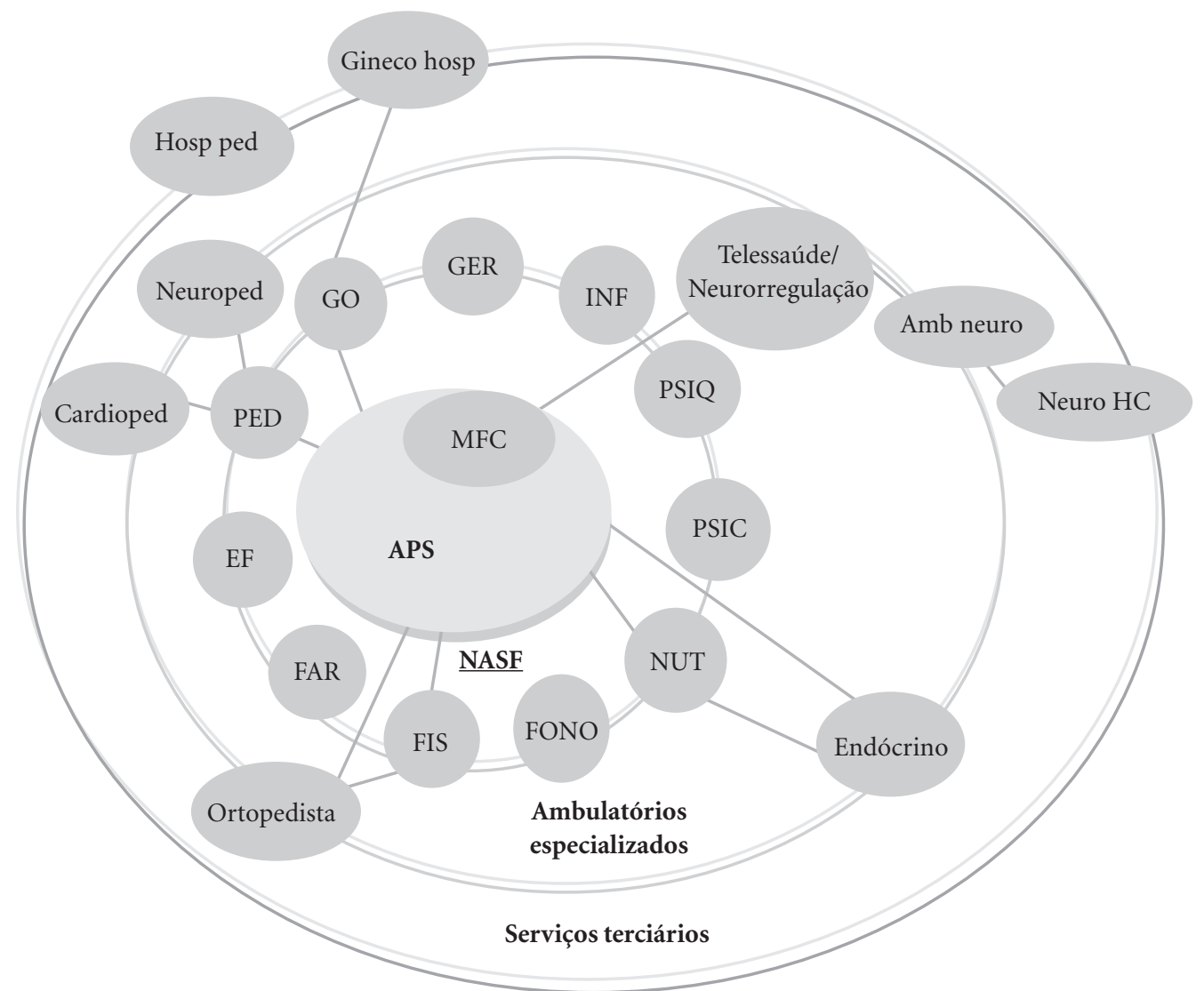

Legenda:

APS= atenção primária à saúde;

FIS= fisioterapeuta;

$\mathrm{MFC}=$ médico de família e comunidade;

$\mathrm{FAR}=$ farmacêutico;

$\mathrm{GO}=$ gineco-obstetra;

$\mathrm{EF}=$ educador físico;

GER=geriatra;

$\mathrm{INF}=$ infectologista;

$\mathrm{PSIQ}=$ psiquiatra;

$\mathrm{PSIC}=$ psicólogo;

NUT $=$ nutricionista;

$\mathrm{FONO}=$ fonoaudiólogo;

Cardioped $=$ cardiopediatra;

Neuroped $=$ neuropediatra;

Telessaúde/Neurorregulação $=$ telerregulação em neurologia;

Neuro HC = neurologia do Hospital das Clínicas;

Gineco hosp = ginecologia hospitalar;

Hosp ped = Hospital pediátrico.

Figura 1. Rede de Atenção especializada à Saúde de Curitiba.

Fonte: elaboração dos autores

atuação regionalizada e descentralizada como os NASF, com contato personalizado com os generalistas que lhes referenciam pacientes e com eles discutem dúvidas, condutas e projetos terapêticos, exercitam regulação negociada e educação permanente mútua. Ou seja, devem realizar matriciamento, o qual tem o potencial de dissolver o problema da referência e contrarreferência, que se hipertrofia e cresce quando os profissionais especializados e generalistas da APS trabalham isolados, não se conhecem, não se conversam, não se respeitam, não colaboram e não aprendem uns com os outros. Em outros termos, esta proposta constitui um possível modus operandi de operacionalizar progressivamente (ou ao menos contribuir substantivamente para) a construção da RAS de forma inteiramente coerente com Men$\operatorname{des}^{9} \mathrm{e}$ as diretrizes federais atuais da RAS ${ }^{10}$. Isso certamente é sustentável, pelo menos no que diz respeito ao cuidado ambulatorial especializado que pode ser relativamente descentralizado (não depende de tecnologias duras e ou de alto custo 
Tabela 1. Filas de espera para especialidades.

\begin{tabular}{lrr}
\hline \multicolumn{1}{c}{ Especialidade } & \multicolumn{1}{c}{ Fila } & \multicolumn{1}{c}{ Fila } \\
jun/14 & jun/15 \\
\hline Clínica Geral Alzheimer & 374 & 456 \\
Clínica Geral Parkinson & 165 & 88 \\
Geriatria Geral & 1.140 & 288 \\
Ginecologia Geral & 10.975 & 7.644 \\
Ginecologia Cirúrgica & 5.433 & 4.420 \\
Ginecologia Reprodução Humana & 1.250 & 882 \\
HIV/AIDS & 396 & 63 \\
Hepatites Virais & 106 & 20 \\
Infectologia Geral & 1.139 & 334 \\
Gastro - Hepato - Vírus & 209 & 164 \\
Psiquiatria Geral & 4.415 & 2.547 \\
Pediatria Geral & 611 & 104 \\
Total & 26.213 & 17.010 \\
\hline
\end{tabular}

Fonte: Relatório Gerencial: Mapa Geral Dinâmico. Secretaria Municipal de Saúde de Curitiba.

que necessitam ficar centralizadas espacialmente por motivos de eficiência e economia de escala), uma parte muito significativa dele. Além disso, as várias possibilidades tecnológicas de informação e comunicação contemporâneas existentes no Brasil (telefone, internet, telessaúde, telemedicina etc.) provavelmente permitem também viabilizar algo como um matriciamento regionalizado, mesmo nos casos de menor possibilidade de descentralização dos especialistas ou seus equipamentos.

A indução financeira federal de equipes de atenção especializada semelhantes aos NASF, como formato organizativo de atenção secundária oficial do SUS, incluindo talvez a maior parte das especialidades médicas, colocaria fim ao pagamento por procedimentos, há décadas vigente no SUS. Significaria um primeiro, importante e tardio movimento de construção institucional da atenção especializada coerente com a RAS; avançando na solução do problema da relação entre APS e especialistas e na estruturação efetiva, coerente e eficiente de uma atenção especializada ambulatorial que de fato seja acessível quando necessária, coordenada pela APS e parceira das equipes da ESF.

\section{Colaboradores}

CD Tesser concebeu e participou de todas as fases da confecção do artigo. P Poli Neto participou da redação inicial de parte do artigo, revisão crítica e bibliográfica e redação final do artigo. 


\section{Referências}

1. Starfield B. Atenção Primária: Equilíbrio entre Necessidades de Saúde, serviços e teconologia. Brasília: Unesco, Ministério da Saúde; 2002

2. Gérvas J, Pérez Fernández M. El fundamento científico de la función de filtro del médico general. Rev. bras. epidemiol. 2005; 8(3):205-218.

3. Brasil. Ministério da Saúde (MS). Secretaria de Atenção à Saúde. Departamento de Atenção à Saúde. Avaliação Normativa do Programa de Saúde da Família no Brasil: monitoramento da implantação e funcionamento das equipes de saúde da família: 2001-2002. Brasília: MS; 2004.

4. Oliveira RC, Correa AA, Ferreira AG, Marques ZFA. A reorganização da atenção secundária como estratégia para a garantia da integralidade. In: Magalhães Júnior HM, organizador. Desafios e inovações na gestão do SUS em Belo Horizonte: a experiência de 2003 a 2008. Belo Horizonte: Mazza Edições; 2010. p. 149-171.

5. Spedo SM, Pinto NRS, Tanaka OY. O difícil acesso a serviços de média complexidade do SUS: o caso da cidade de São Paulo, Brasil. Physis 2010; 20(3):953-972

6. Brasil. Ministério da Saúde (MS). Secretaria de Atenção à Saúde. Departamento de Atenção Básica. Núcleo de Apoio à Saúde da Família. Brasília: MS; 2014. (Cadernos de Atenção Básica, n.39). [acessado 2016 fev 15]. Disponível em: http://bvsms.saude.gov.br/bvs/publicacoes/nucleo_apoio_saude_familia_cab39.pdf

7. Tesser CD. Núcleos de Apoio à Saúde da Família, seus potenciais e entraves: uma interpretação a partir da atenção primária à saúde. Interface (Botucatu) [Internet]. [cited 2017 Jan 11]. Available from: http:// www.scielo.br/scielo.php?script=sci_arttext\&pi$\mathrm{d}=\mathrm{S} 1414-32832016005024104 \& \operatorname{lng}=\mathrm{en}$. In press 2016. Epub Nov 03, 2016. http://dx.doi.org/10.1590/180757622015.0939 .

8. Cunha GT, Campos GWS. Apoio Matricial e Atenção Primária em Saúde. Saude soc. 2011; 20(4):961-970.

9. Mendes EV. As redes de atenção à saúde. Brasília: Organização Pan-Americana da Saúde; 2011.

10. Brasil. Ministério da Saúde (MS). Portaria no 4.279, de 30 de dezembro de 2010. Estabelece diretrizes para a organização da Rede de Atenção à Saúde no âmbito do Sistema Único de Saúde (SUS). Diário Oficial da União 2010; $31 \mathrm{dez}$.

11. Jimenez E, Simão MBG, Shimazaki ME. Programa mãe curitibana: avançando na humanização da atenção materno-infantil. Divulg saúde debate 2003; 26:21-29.

12. Marques EC. Notas críticas à literatura sobre Estado, políticas estatais e atores políticos. Rev. bras. Ci. Soc. 1997; 43:67-102.

13. Pires MRGM, Demo P. Políticas de saúde e crise do Estado de Bem-Estar: repercussões e possibilidades para o Sistema Único de Saúde. Saúde Soc. 2006; 15(2):56-71.

14. Rodrigues JH. Conciliação e reforma no Brasil: um desafio histórico-cultural. São Paulo: Editora Nova Fronteira; 1982.

15. Santos MAB, Gerschman S. As segmentações da oferta de serviços de saúde no Brasil - arranjos institucionais, credores, pagadores e provedores. Cien Saude Colet 2004; 9(3):795-806.
16. Scheffer M, Biancarelli A, Cassenote A, coordenadores. Demografia Médica no Brasil: dados gerais e descrições de desigualdades. São Paulo: Conselho Regional de Medicina do Estado de São Paulo, Conselho Federal de Medicina; 2011.

17. Campos CEA. As origens da rede de serviços de atenção básica no Brasil: o Sistema Distrital de Administração Sanitária. Hist Ciênc Saúde 2007; 14(3):877-906.

18. Buss PM, Carvalho AI. Desenvolvimento da promoção da saúde no Brasil nos últimos vinte anos (1988-2008). Cien Saude Colet 2009; 14(6):2305-2316.

19. Teixeira CF, Solla JP. Modelo de atenção à saúde no SUS: trajetória do debate conceitual, situação atual, desafios e perspectivas. In: Lima NT, Gerchman S, Edler FC, organizadores. Saúde e democracia: história e perspectivas do SUS. Rio de Janeiro: Editora Fiocruz; 2005. p. 451-477.

20. Tesser CD, Norman AH. Repensando o acesso ao cuidado na Estratégia Saúde da Família. Saude soc. 2014; 23(3):869-883.

21. Bahia L. As contradições entre o SUS universal e as transferências de recursos públicos para os planos e seguros privados de saúde. Cien Saude Colet 2008; 13(5):1385-1397.

22. Mendes A, Weiller JAB. Renúncia fiscal (gasto tributário) em saúde: repercussões sobre o financiamento do SUS. Saúde debate 2015; 35(105):491-505.

23. Campos GWS. Equipes de referência e apoio especializado matricial: um ensaio sobre a reorganização do trabalho em saúde. Cien Saude Colet 1999; 4(2):393-403.

24. Campos GWS, Domitti AC. Apoio Matricial e Equipe de Referência: uma metodologia para gestão do trabalho interdisciplinar em saúde. Cad Saude Publica 2007; 23(2):399-408.

25. Campos GWS, Figueiredo MD, Pereira Junior N, Castro CP. A aplicação da metodologia Paideia no apoio institucional, no apoio matricial e na clínica ampliada. Interface (Botucatu) 2014; 18(Supl. 1):983-995.

26. Oliveira MM, Campos GWS. Apoios matricial e institucional: analisando suas construções. Cien Saude Colet 2015; 20(1):229-238.

27. Giovanella L. A atenção primária à saúde nos países da União Européia: configurações e reformas organizacionais na década de 1990. Cad Saude Publica 2006; 22(5):951-963.

28. Gérvas J, Rico A. La coordinación en el sistema sanitario y su mejora a través de las reformas europeas de la Atención Primaria. SEMERGEN 2005; 3(9):418-423.

29. Ojeda JJ, Freire JM, Gérvas J. La coordinación entre atención primaria y especializada: reforma del sistema sanitario o reforma del ejercicio profesional. Revista de Administración Sanitaria 2006; 4(2):357-382.

30. Almeida PF, Giovanella L, Mendonça MH, Escorel S. Desafios à coordenação dos cuidados em saúde: estratégias de integração entre níveis assistenciais em grandes centros urbanos. Cad Saude Publica 2010; 26(2):286-298.

31. Bower P, Gilbody S, Richards D, Fletcher J, Sutton A. Collaborative care for depression in primary care. Making sense of a complex intervention: systematic review and meta-regression. Br J Psychiatry 2006; 189:484-493. 
32. Vingilis E, Paquete-Warren J, Kates N, Crustolo A, Greenslade J, Newman S. Descriptive and process evaluation of a shared primary care program. Internet $J \mathrm{Al}$ leid Health Sci Pract 2007; 5(4):1-10.

33. Kates N, Craven M. Collaborative Working Group of the College of Family Physicians of Canada, Canadian Psychiatric Association. Shared mental health care. Update from the Collaborative Working Group of the College of Family Physicians of Canada and the Canadian Psychiatric Association. Can Fam Physician 2002; 48:936.

34. Lythgoe MP, Abraham S. Good practice in shared care for inflammatory arthritis. Br J Gen Pract 2016; 66(646):275-277

35. Kelly BJ, Perkins DA, Fuller JD, Parker SM. Shared care in mental illness: A rapid review to inform implementation. Int J Ment Health Syst 2011; 5:31.

36. Foy R, Hempel S, Rubenstein L, Suttorp M, Seelig M, Shanman R, Shekelle PG. Meta-analysis: effect of interactive communication between collaborating primary care physicians and specialists. Ann Intern Med 2010; 152(4):247-258

37. Terraza Núñez R, Vargas Lorenzo I, Vázquez Navarrete ML. La coordinación entre niveles asistenciales: una sistematización de sus instrumentos y medidas. Gac Sanit 2006; 20(6):485-495.

38. Almeida PF, Gervas J, Freire JM, Giovanella L. Estratégias de integração entre atenção primária à saúde e atenção especializada: paralelos entre Brasil e Espanha. Saude Debate 2013; 37(98):400-415.

39. Florianópolis. Prefeitura Municipal. Secretaria Municipal de Saúde. Protocolo de atenção em saúde mental Município de Florianópolis. Tubarão: Copiart; 2010. [acessado 2016 fev 15]. Disponível em: http://www.pmf.sc. gov.br/arquivos/arquivos/pdf/05_08_2011_9.41.44.1bf62fa463bec5495279a63c16ed417f.pdf

40. Saraiva SAL, Cremonese E. Implantação do modelo de apoio matricial em saúde mental no município de Florianópolis - SC. In: Brasil. Ministério da Saúde (MS). Secretaria de Atenção à Saúde. Departamento de Atenção Básica. III Concurso Nacional de Experiências em Saúde da Família: trabalhos premiados. Brasília: MS; 2008. p. 39-50.

41. Frosi RV, Tesser CD. Práticas assistenciais em saúde mental na atenção primária à saúde: análise a partir de experiências desenvolvidas em Florianópolis. Cienc Saude Colet 2015; 20(10):3151-3161.

42. Pavan C, Trajano ARC. Apoio institucional e a experiência da Política Nacional de Humanização (PNH) na Freguesia do Ó, Brasilândia, São Paulo, Brasil. Interface (Botucatu) 2014; 18(Supl. 1):1027-1040.

43. Oliveira G. Devir apoiador: uma cartografia da função apoio [tese]. Campinas: Universidade Estadual de Campinas; 2011.

44. Delfini PSS, Sato MT, Antoneli PP, Guimarães POS. Parceria entre CAPS e PSF: o desafio da construção de um novo saber. Cien Saude Colet 2009; 14(Supl. 1):1483-1492.

45. Dimenstein M, Severo AK, Brito M, Pimenta AL, Medeiros V, Bezerra E. O apoio matricial em Unidades de Saúde da Família: experimentando inovações em saúde mental. Saude soc 2009; 18(1):63-74.
46. Figueiredo MD, Campos RO. Saúde Mental e Atenção Básica à Saúde: o apoio matricial na construção de uma rede multicêntrica. Saúde Debate 2008; 32(78-80):143149.

47. Pinto AGA, Jorge MSB, Vasconcelos MGF, Sampaio JJC, Lima GP, Bastos VC, Sampaio HAC. Apoio matricial como dispositivo do cuidado em saúde mental na atenção primária: olhares múltiplos e dispositivos para resolubilidade. Cien Saude Colet 2012; 17(3):653-660.

48. Quindere PHD, Jorge MSB, Nogueira MSL, Costa LFA, Vasconcelos MGF. Acessibilidade e resolubilidade da assistência em saúde mental: a experiência do apoio matricial. Cien Saude Colet 2013; 18(7):2157-2166.

49. Hirdes A, Scarparo HBK. O labirinto e o minotauro: saúde mental na Atenção Primária à Saúde. Cien Saude Colet 2015; 20(2):383-393.

50. Moyses ST, Silveira Filho AD, Moysés SJ, organizadores. Laboratório de inovações no cuidado das condições crônicas na APS: A implantação do modelo de atenção às condições crônicas na UBS Alvorada em Curitba. Brasília: OPAS/CONASS; 2012. [acessado 2015 ago 25]. Disponível em: http://www.saude.curitiba.pr.gov.br/ images/programas/livor_estudo-de-caso-alvorada.pdf

51. Massuda A, Poli Neto P, Drehmer V. A Atenção Primária à Saúde (APS) no SUS Curitiba: um desafio para o Brasil, passar de uma APS programática para uma mais acessível e abrangente. In: Souza MF, Machado MS, Mendonça AVM, organizadores. Saúde da Família nos Municípios Brasileiros: os reflexos dos 20 anos no espelho do futuro. Campinas: Saberes; 2014. p. 121-177.

52. Curitiba. Prefeitura Municipal. Secretaria Municipal de Saúde. Novas possibilidades de organizar o acesso e a agenda na Atenção Primária à Saúde. 2014. [acessado 2016 fev 15]. Disponível em: http://www.saude.curitiba.pr.gov.br/images/cartilha\%20acesso\%20avan\%C3\%A7ado\%2005_06_14.pdf

53. Curitiba. Prefeitura Municipal. Secretaria Municipal de Saúde. Carteira de Serviços - guia para profissionais de saúde. Relação de serviços e condições abordadas na APS. SMS; 2013. [acessado 2016 fev 15]. Disponível em: http://www.saude.curitiba.pr.gov.br/images/Manual \%2014abr14.pdf Acesso: 15 fev 2016

54. Curitiba. Decreto $n^{\circ} 560,28$ de março de 2013. Dispõe sobre a regulamentação da Atenção Primária à Saúde na Secretaria Municipal da Saúde. Diário PR 2013; 2 abr.

55. Curitiba. Instrução Normativa $n^{\circ} 1$. Estabelece diretrizes sobre o processo de trabalho dos profissionais dos NASF no município de Curitiba. Diário PR 2014; 16 jan.

56. Massuda A. Construindo novos caminhos para a reforma na saúde: reflexões sobre a práxis da formulação e implementação de mudanças na gestão e no modo de produção de práticas de saúde no SUS em Curitiba [tese]. Campinas: Universidade Estadual de Campinas; 2014.

Artigo apresentado em 15/02/2016

Artigo aprovado em 19/08/2016

Versão final apresentada em 21/08/2016 
